Article preprint

\title{
Modelling Distance Measurement Equipment (DME) signals interfering an airborne GNSS receiver
}

This is the peer reviewed version of the following article: Modelling Distance Measurement Equipment (DME) signals interfering an airborne GNSS receiver, which has been accepted for publication in final form in the Journal of Navigation and can be found in the final version at https://www.ion.org/publications/browse.cfm.

It will be published in the summer issue of NAVIGATION, Journal of the Institute of Navigation, Vol. 65, No. 2. 2018

If you want to cite this article: Please use:

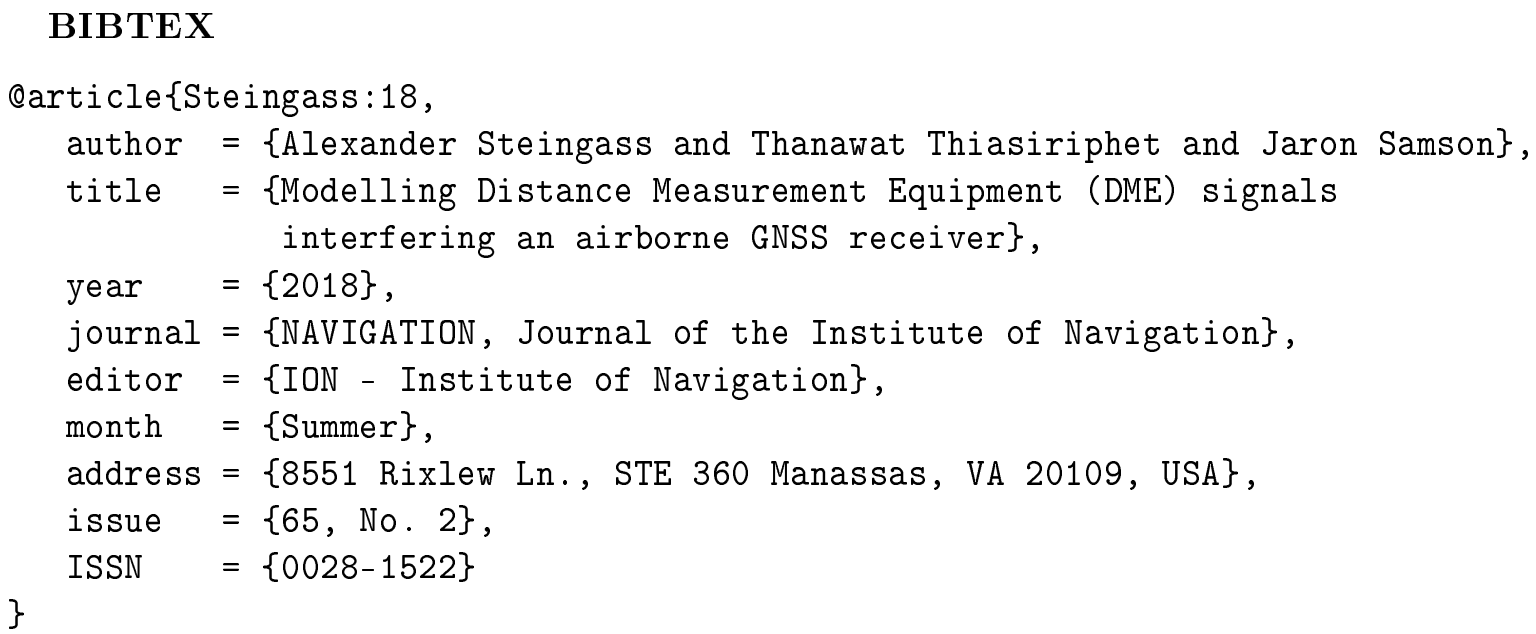

\section{Plaintext}

Steingass, Alexander and Thanawat, Thiasiriphet and Samson, Jaron (2018)

Modelling Distance Measurement Equipment (DME) signals interfering an airborne GNSS receiver.

Navigation, Journal of the Institute of Navigation, 8551 Rixlew Ln., STE 360 Manassas, VA 20109, USA.

Institute of navigation.

Issue 65, No. 2, summer 2018.

ISSN $0028-1522$

Please note: The model download is currently under construction. It will be available soon - at least when the paper is published. 


\title{
Modelling Distance Measurement Equipment (DME) signals interfering an airborne GNSS receiver
}

\author{
Alexander Steingass ${ }^{* 1}$, Thanawat Thiasiriphet ${ }^{\dagger 1}$ and Jaron Samson ${ }^{\ddagger 2}$ \\ ${ }^{1}$ German Aerospace Center - DLR \\ ${ }^{2}$ European Space Agency - ESA
}

March 1, 2018

\section{Abstract}

This publication describes an end-to-end model to generate Distance Measurement Equipment (DME) signals as an interference source to airborne Global Satellite Navigation Systems (GNSS).

Both satellite navigation systems, the Global Positioning System (GPS) and GALILEO, use the lower L-band ${ }^{\square}$ for wideband navigation services and are sharing the same frequency band with DME. Any GNSS Receiver operating in the mentioned bands will receive DME signals and will have to deal with them as interference. This publication describes a model to rebuild the measured DME signals at the receiver input to allow simulations of the interference effect. Prior to this work we only found models based on propagation estimation. No

\footnotetext{
*alexander.steingass@dlr.de

$\dagger$ thanawat.thiasiriphet@dlr.de

†jaron.samson@esa.int

${ }^{1}$ GALILEO: E5 1164-1215 MHz,

GPS L5 1164-1189 MHz
}

model existed which is based on real world measurements of DME signals. Thus, the German Aerospace Center (DLR) has carried out a flight measurement campaign at the European DME hotspot near Frankfurt (Main), Germany. From the data of the measurement campaign we have developed the new model. This measurement based model is much more accurate than the existing models since it accounts for the propagation and the DME transmission and the GNSS receiver antenna effects. We provide this model to the community to allow a more realistic forecast of the DME-GNSS interference situation.

\section{Introduction}

During the definition phase of the European satellite navigation system GALILEO, it was obvious to use the L1 band for the core part of the system. The use of L1 was mainly based on the requirement to be compatible with the GPS system. To fa- 
cilitate the advanced civil receivers an autonomous ionosphere correction a second frequency band was needed. As the L band is densely allocated, the choice was made to use the $E 5^{\square}$ band. Especially for civil aviation a band with a protected bandwidth was necessary. The E5a band was selected since this band already had a frequency protection namely for the distance measuring equipment DME [2].

The DME system radiates short twin pulses each of a duration of $6 \mu$ s and with a power of up to $2 \mathrm{~kW}$. The DME system allows to estimate the distance from the aircraft to a ground station. In order that GALILEO or GPS can coexist with the DME system in the E5 band such GNSS receivers have to be robust against DME interference.

Previous publications [17, 15, 16, 6] propose theoretically based models to simulate the DME signal strength at the GNSS antenna output of the aircraft.

The basic approach to mitigate the DME signal is blanking the received pulses by shutting down the antenna input during a pulse. This blanking approach reduced the interference of the DME signal on the one hand but reduces as well the power of the satellite signal. Already [12] came to the conclusion that the "(DME) Power level is important" but the DME power level is only a theoretical estimation at the moment. [8, 27, 201, 1.9] underlined the importance of future measurements.

Only a very small number of publications are based on real world measurements or experiments [24, 2.9]. In these publications real world GPS signals and DME signals

\footnotetext{
${ }^{2} 1164-1214 \mathrm{MHz}$
}

had been measured together by using GPS receivers which had been modified to apply signal blanking techniques for DME signal mitigation. The GPS receiver had been mounted in an aircraft flying over an European test area. As a result we get real world measurement, which is important but only valid for a GPS signal and the selected receiver using the selected blanking method. A generic, GNSS signal and receiver independent measurement based interference model is still missing.

In general there is a lack of location and altitude dependent measurement data for strength and pulse load of received DME signals. Therefore the German Aerospace Centre (DLR) conducted a detailed measurement campaign in 2009 where the DLR team recorded the received GNSS signals and their interference in the L1 and E5 band during flight tests. This experiment has been published [30]. Recorded signal snapshots have been used to estimate the interference effects on GALILEO receivers [3] by regenerating the DME signal with an arbitrary wave form generator (AWG) and overlaying the DME signal with a GALILEO signal that had been generated by a hardware GALILEO signal generator.

After these tests, a systematic data evaluation has been carried out, which results in a generic DME interference model, which is capable to generate an interference scenario from the ICAO COM3 [22] database and which is verified against the measurements. The successful verification offers a validated model to the navigation community.

This article publishes the verified model which rebuilds the DME signals as an interference situation at a GNSS antenna of 
an aircraft. Although it is relatively easy to measure the pattern of a GNSS antenna on a test stand it is difficult to measure the antenna diagram while the antenna is mounted on the aircraft, taking into account the effect of the metal fuselage. Our evaluation of the recorded DME signals together with geometric information such as position, roll pitch an yaw of the aircraft, allowed us to derive an antenna - fuselage model. In contrast to other theory based DME interference models [33, 28] that do not consider the GNSS antenna and fuselage effects our model is measurement based and includes the aircraft fuselage.

Considering the DME ground as well as the GNSS aircraft antenna the new DME interference model allows an end-to-end simulation from the DME transmitters to the aircraft GNSS antenna output.

\section{Propagation Effects}

Precise modelling requires precise propagation estimation. In Europe the most dense DME hotspot has been estimated near Frankfurt (Main) in Germany at the way point RUDUS ${ }^{[3]}$ [25]. From transmitter antenna input to receiver output the following attenuations and gains are relevant:

\section{Radio Horizon}

The radio horizon $D$ is the minimum distance from the aircraft to a DME station beyond which no reception was possible. In order to model diffraction of radio waves around the Earth surface many publications [10] corrected the radio hori- zon by calculating an "effective Earth radius" that equals usually $\frac{4}{3}$ times the physical radius of the Earth. This approach leads to higher distances from where stations can be received. In our measurements we found a good match between our measured radio horizon and the radio horizon being calculated with the physical Earth radius. Thus we conclude that diffraction is not applicable in this case, likely due to the high RF frequencies in L-band.

To calculate the radio horizon let $r_{e}$ be the physical radius of the Earth, $h_{a}$ the altitude of the aircraft above mean sea level (MSL) and $h_{s}$ the height of the station above MSL, then we found

$$
D=\sqrt{\left(r_{e}+h_{a}\right)^{2}-r_{e}^{2}}+\sqrt{\left(r_{e}+h_{s}\right)^{2}-r_{e}^{2}}
$$

Equation $\mathbb{0}$ can be reformulated to:

$$
D=\sqrt{h_{a}^{2}+2 r_{e} h_{a}}+\sqrt{h_{s}^{2}+2 r_{e} h_{s}}
$$

as the radio horizon $D$.

Assuming a receiver on the sea surface $\left(h_{a}=0\right)$ equation $\square$ results in:

$$
D=\sqrt{h_{s}^{2}+2 r_{e} h_{s}}
$$

Since the altitude of the station is much less than the radius of the earth, equation [3] can be approximated to:

$$
D \approx \sqrt{2 r_{e} h_{s}}
$$

This equation is well known as maritime radio horizon [IT].

\footnotetext{
${ }^{3} 50^{\circ} 2^{\prime} 51.32^{\prime \prime} \mathrm{N}, 8^{\circ} 4^{\prime} 41.77^{\prime \prime} \mathrm{E}$
} 


\section{Transmitter Antenna}

Nearly all DME stations in Germany are using the Kathrein "FAN 86" DME Antenna [18]. Thus we use the FAN 86 antenna model for all transmitting stations. The FAN 86 consists of vertically stacked dipole antennae, which are powered by a phase shift array to achieve the maximum power density at an elevation of $5^{\circ}$ as specified in [2:3]. Every single of the used dipole antennas shows a null ${ }^{\mathbb{T}}$ at the zenith. Therefore the combination of these dipole antennas also shows a null pointing to the zenith.

\section{Terrain Attenuation}

We have observed strong attenuations by hills and mountain chains such as the Alps ${ }^{\underline{1}}$. Wherever a DME station was optically invisible by terrain, a reception was impossible. One reason why RUDUS is a hot spot is that for aircraft position more south than RUDUS in Germany many stations in or behind the Alps are blocked. At RUDUS the radio horizon is located just in front of the Alps for an aircraft being at $40.000 \mathrm{ft}$ MSL. [31] provides propagation curves for L-band at $1200 \mathrm{MHz}$. The most important outcome of [31] is that the propagation loss equals the free space loss as long as the station is optically visible. Behind the horizon the propagation loss drops so rapidly below any relevant detection level that we can neglect these stations.

\section{Free Space Loss}

The free space loss $G_{F S L}$ is well known with $G_{F S L} \propto \frac{1}{D^{2}}$. Attenuations by atmo-

\footnotetext{
${ }^{4} \mathrm{~A}$ null of an antenna is the direction from where no signal can be received.

${ }^{5}$ Central European mountain chain
}

spheric effects and gases are not relevant for the E5 frequency band.

\section{Aircraft Antenna}

Although the pattern of the antenna itself is known in detail (compare [21], Page 15 Figure 5 et. al. or [26], Page 12 Figure 3) when installing the antenna on the aircraft fuselage, the pattern of the combined antenna-fuselage changes dramatically, especially in the lower hemisphere, due to near field interaction between the fuselage and antenna. In the considered scenario we are generating a DME signal as interference source to GNSS. Thus, we have used a typical dual frequency satellite navigation patch antenna for the measurement which was mounted on the top of the aircraft near the cockpit pointing towards the sky for best satellite reception. This antenna location is very different from the location of DME reception antennas that are placed below the fuselage of the aircraft to have best DME reception.

For interference scenarios often a "straight and levelled" flight is assumed for a typical scenario [3.3] or the aircraft antenna and its orientation is neglected at all [28]. We see the necessity for accurate modelling of the aircraft's attitude to represent relevant scenarios.

For evaluating the measurement data, we used the ID sequence of the DME ground stations to identify to which DME stations the received pulses belong to. This assignment worked well even though a lot of DME pulses had been received simultaneously. During the measurement the flight management system (FMS) and the data recording had been synchronized by time stamping 
both data streams. From the detected ID of the DME station we determined its location from the COM3 database [22]. Taking into account the received power of ID pulses together with aircraft's attitude (from gyroscopes of FMS) and position data (FMS) and the DME ground station location we were able to determine the aircraft antenna gain for every specific transmission coming from a specific direction. Eventually considering all available measurements we were able to calibrate the antenna diagram for a typical GNSS antenna that is interfering with the aircraft its mounted on.

Figure 1(a) shows the result of this evaluation where each spot shows the median of all ID pulse receptions. Please recall that the median and the mean are identical assuming a Gaussian distribution. In Figure 1(a) the aircraft nose is at $\left(\mathrm{az}=0^{\circ}\right.$, elev $=$ $0^{\circ}$.

The standard manoeuvre for an aircraft is the two minute turn [I] which results in a banking angle of about $30^{\circ}$ for our measurement aircraft. The dashed lines in Figures 1(a) and 1(b) are showing the horizon line for $40^{\circ}$ banking. Below the horizon line the DME signal was picked up seldom. This might be surprising at the first hand since we did fly straight over the stations, but precise calculations confirmed that a small deviation of the aircraft from the stations zenith results in a big elevation derivation from the horizon. Furthermore due to the null at the Zenith the antenna is showing a cone shaped area where no reception can occur. We used the seldom contacts to DME stations in the lower hemisphere and calculated an average gain of $-15 \mathrm{~dB}$. With this gain we filled all data points where we had no reception.

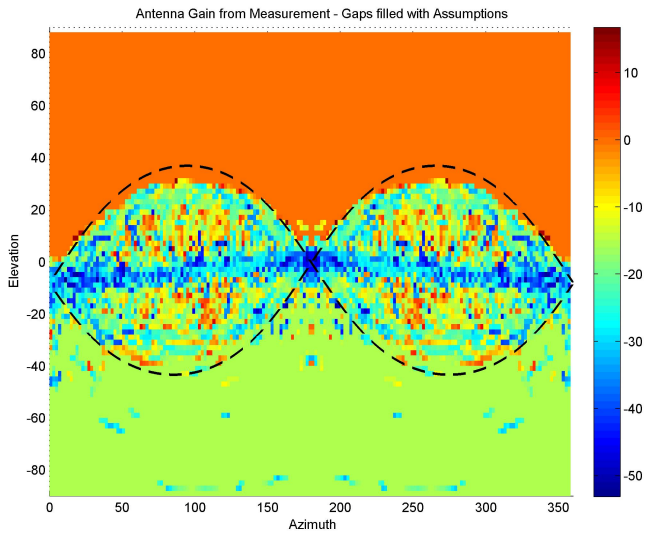

(a) Raw data from measurement

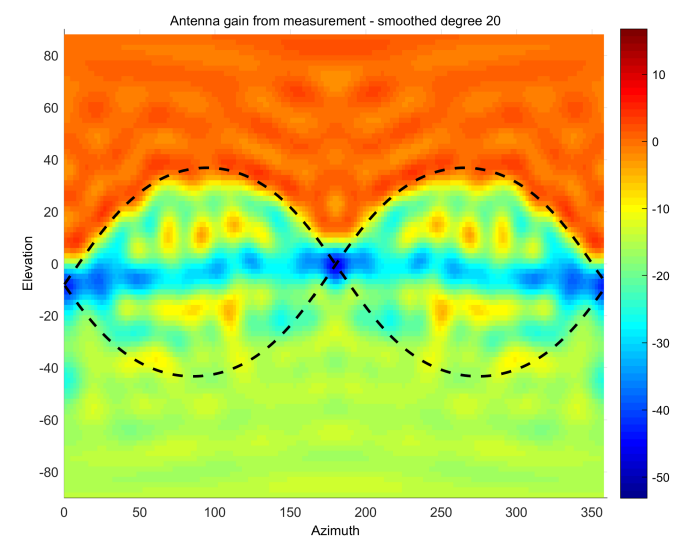

(b) Smoothed by sperical harmonics degree 20

Figure 1: Antenna pattern derived from measurements - Gain in dB 
Since DME stations are situated on the ground we have no gain information in the upper hemisphere. Therefore we filled this area with samples representing $0 \mathrm{~dB}$ that result in the orange colour.

The high angular resolution of data points is showing a quite scattered Figure. To smooth the angular data and still account for every data point we followed the spherical harmonics analysis [32]. The spherical harmonics approach determines a series of coefficients by projecting the original data onto spherical harmonic functions. These represent a complete series of orthonormal functions. An important property is that the coefficients are decreasing with raising degree. So they can be used as a smoothing filter by setting higher coefficients to zero.

In Figure 1(b) we have applied the spherical harmonic method. Now a smoother antenna pattern can be seen. The most important feature of Figure 1(b) is the finding that the antenna gain at the horizon is significantly attenuated (blue belt at the horizon) between 30 to $40 \mathrm{~dB}$. Referring again to the often assumed "straight and levelled" flight [33] it is clear that the real antenna pattern plays a significant role since nearly all stations are received from the horizon, thus most of the stations are significantly attenuated.

This strong attenuation at the horizon for a fuselage mounted antenna can also be found in other publications even if other frequencies, antenna types and aircraft types are used [14] (Figure 5 and 6), [1:3] (Figure 13 and 15). Thus, we conclude that the strong horizon attenuation is inflicted by the aircraft fuselage. Thus the smoothed antenna diagram should show this attenua- tion effect as well.

Also an important finding is the antenna gain from stations below the horizon line: Here we have measured an attenuation of 20 to $25 \mathrm{~dB}$.

We can see fluctuations in the region above and below the levelled horizon line between the dashed lines. We assume that these high and low spots are generated by constructive and destructive interference from the direct path and paths reflected by the fuselage or even refracted paths. Revisiting [13] (Figure 13) and [14] (Figure 4 and 5) show similar fluctuations as we see in Figure 1(b). Thus a smoothed diagram should show this fluctuation effect as well.

Baring in mind these two effects we can now adapt the smoothing level using the spherical harmonic functions for the measurement antenna in a way, that these fluctuations are well visible and the scatter effects caused by the high resolution disappear. By comparing different smoothing levels we came to the result, that a smoothing level of 20 as shown in Figure 1(b) fulfils these requirements very well. Thus we recommend this degree of smoothing for the antenna.

Beside proper representation of the aircraft antenna we conclude that the orientation of the aircraft plays a major role. With the real antenna pattern the critical scenarios can be identified and simulated precisely.

With these new findings we see the last turn to the final approach as such an exemplary scenario. In the final approach phase the GNSS requirements are much higher than for an en route scenario and the banking brings potential DME stations into enhanced view. Further investigations should 
clarify if there might be other flight scenarios where a worse interference situation may occur than at the hot spot RUDUS.

\section{The Model}

The developed DME software model is capable of generating DME/TACAN signals at the aircraft's antenna output from a user selected DME/TACAN constellation at an assumed aircraft position at a selected altitude and attitude. In this paper, the term 'DME stations' shall represent both DME and TACAN stations. When the model is used for simulations, a list of visible DME stations can be generated, from which a list of received DME pulses and a baseband DME signal can be created as the output from the proposed software model. DME pulses, their shape and duration are generated following the Minimum Operational Performance requirements procedures (MOPS) [7] for DME. ID sequence pulses and squitter pulses are generated following the DME MOPS [7]. Reply pulses are created as randomly generated squitter pulses. TACAN main and auxiliary bursts are considered but the TACAN double sinusoidal amplitude modulation is not yet implemented in the current version of the software model.

For simulation, the user can define a static aircraft location and attitude. Alternatively time series of location and attitude together with timestamps representing a flight path can be used. We used the ICAO COM3 ground station database [22] for the simulations presented in this paper. The information on the location, peak power of DME ground stations are given in the COM3 database, however, the station height information is not available in the COM3 database. Thus the height of all ground stations has been assumed to be at MSL for the following simulations. Terrain effects are not implemented in the current version of the software model, therefore terrain attenuation, shadowing and ground multipath reflections are not included in the simulation results. Setting all DME stations at MSL will then result in a scenario where we expect a high pulse load from DME ground stations, which will be a bad case for GNSS receivers.

The DME software model is divided into three modules: Geometric module (GM), Pulse Generation module (PG) and Pulse Modulation module (PM). The three provided modules can be used independently by the user for his own purposes.

An overview of the DME software model is shown in Figure 2 .

\section{Geometric Module: GM}

An artificial scenery is internally created in the GM module from a selectable ground station database taking into account an aircraft position, altitude and attitude. Internal values such as received power from each DME station, distances between aircraft and each DME ground station as well as DME station visibility are calculated from the used artificial scenery. The GM module outputs a list of visible DME ground stations and their parameters respectively. The GM module is only dependent on the geometry which leads always to the same result for the same geometric constellation. A time series of the visible DME list can be generated by updating the input param- 


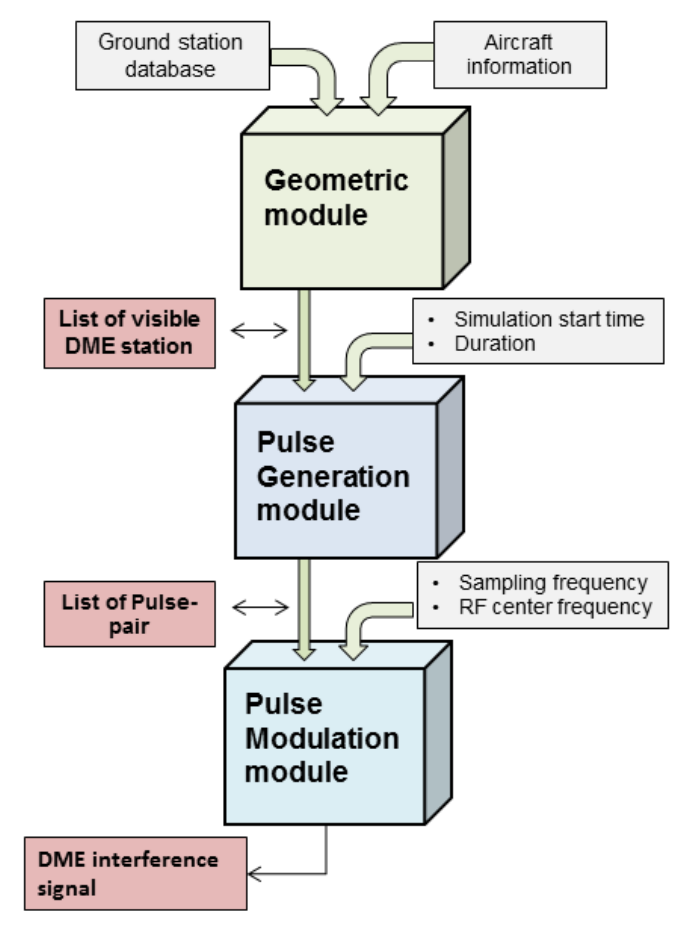

Figure 2: Overview of DME software modules

eters of the created artificial scenery at different aircraft positions, altitudes and attitudes with corresponding time stamps.

\section{Pulse generation Module: PG}

The PG module requires a list of DME visible stations (usually the output from the GM module) and outputs a list of DME pulse-pairs seen at the aircraft antenna output in a given time duration. The generated list contains information of each DME pulse pair such as power, frequency, phase and time. The PG module is fully time dependent. Starting time and length of the simulation is set by the user as an input parameter.

\section{Pulse modulation Module: PM}

The PM module is used to create a baseband discrete-time, sampled DME signal from a list of DME pulse-pairs (usually the output from the PG module). The user selects the sampling frequency and centre radio frequency (bandpass centre frequency) of the signal to be generated.

The main idea of dividing the DME model into modules is to give the user flexibility for simulations. For example: If the simulation is only intended to simulate the visibility of the DME ground stations, solely the GM module is needed. If the user is looking for a pulse statistic the output from the GM module and the PG module are to be chosen. Finally using the full simulation chain of GM, PG and PM module will result in a fully sampled RF signal ready to be used with an arbitrary signal generator (AWG).

The coherent time simulation concept for the PG module opens the door for different types of simulations: For example, a statistical DME signal analysis at one aircraft location can be performed by varying only the starting time of the PG module. Another example is the simulation of two different aircraft positions with the same starting simulation time. In this case, the two simulated aircraft positions will result in the same pulse-pairs with different propagation parameters.

The received DME signal at the antenna output can be simulated in time segments to prevent an overload of the computer's memory. For example, a flight of one hour can be partially simulated in blocks of $3 \mathrm{~ms}$ of DME received signal, which is then gen- 
erated every $30 \mathrm{~s}$.

Finally, the DME baseband signal generated by the PM module can be uploaded to a hardware signal generator for radio frequency signal generation. In the software we provide an interface for an Agilent E8267D AWG signal generator.

\section{Results and Compari- son}

We developed a software model which can generate the received DME signal at the output of the aircraft antenna from a DME database taking into account the aircraft position, altitude and attitude. The developed DME software model will be verified by comparing its output to the flight measurement data.

For this comparison we generated the DME signal for the aircraft at the location RUDUS at flight level FL390 ${ }^{\mathbf{E}} . \quad(\approx$ $11.88 \mathrm{~km})$ and FL50 $(\approx 1.52 \mathrm{~km})$. The public ICAO COM3 database [22] was used for determining the ground station parameters. The heights of the stations are not included in the COM3 database, thus all stations were assumed to be located at MSL. The Kathrein FAN 86 DME Antenna [18] was used as the DME ground antenna model. The aircraft antenna diagram smoothed by sperical harmonics degree 20 as shown in Figure 1(b) was selected as the aircraft antenna model. The radio horizon was calculated using equation 2 with an assumption that the ground stations are located at

\footnotetext{
${ }^{6} \mathrm{FL}$ denotes the 'flight level' which is defined as a vertical altitude at standard pressure, nominally expressed in hundreds of feet.
}

MSL. The simulation was performed only for the DME stations transmitting in the E5 band. In the following we will use two criteria for the comparison: Visibility of DME ground stations from the aircraft and a comparison of the received DME signal at the aircraft.

\section{Visibility Comparison}

As the first quality criteria we compare the visibility of DME ground stations, limited by the radio horizon between our model and the measurements.

For the simulation, we determine the pure visibility of the DME ground stations according to the radio horizon regardless of the power level of the received DME signals. In this case, only the aircraft position is influencing the DME ground station visibility. The aircraft orientation is not influencing the visibility of the DME station, since it leads only to an additional attenuation of the received DME signal. The visibility is an output parameter generated by the GM module.

The number of visible stations at the location RUDUS for FL390 and FL50 from the measurement and the simulation are summarized in Table [. For FL50, the number of 11 visible stations from the measurement and the simulation are matched perfectly. For FL390, the measurement data shows that 69 DME stations had been visible at the aircraft while the result from the model shows that 78 stations should be visible.

The visibility of the DME ground stations for FL390 observed in the flight measurement data and at the output from the DME software model are shown in Figure [3. 
Table 1: Number of visible stations at the location RUDUS for FL390 and FL50 from the measurement and the simulation.

\begin{tabular}{|l|c|c|}
\hline & FL390 & FL50 \\
\hline Measurement & 69 & 11 \\
\hline Simulation & 78 & 11 \\
\hline
\end{tabular}

The aircraft location is marked with a 'plus' in the middle of the figures. The radio horizon calculated with equation 2 is visualized as a dashed circle. The visible DME stations are marked with blue dots while the non-visible stations are marked with red crosses.

Some stations located within the radio horizon had not been visible in the measurement data recorded at the aircraft. This effect is most likely caused by terrain attenuation and shadowing. For example, a TACAN station 'SDI' located in SaintDizier, France had not been visible in the measurement data that had been received at RUDUS but this station is located within the calculated radio horizon. The SDI station is located at a height of $150 \mathrm{~m}$ above MSL. Between the SDI station and the measurement aircraft at RUDUS the Hunsrück, which is a hill chain with altitude up to $816 \mathrm{~m}$ above MSL, can be found. Therefore the signal from the SDI station had been blocked by the terrain during the measurement. This effect was not rebuilt by the model since no terrain model was implemented.

Another model deviation is, that DME stations beyond the calculated radio horizon can be visible in the measurement data but not in the model. This happens if DME stations are located higher than MSL which is a mismatch between the real and modelled height. A notable example is the DME station 'CVA' located in Switzerland, on the Piz Corvatsch mountain with a height of $3296 \mathrm{~m}$ above MSL. The model result shows this station as invisible seen from RUDUS, since it is located behind the radio horizon, but it had been visible in the measurements because of its exposed location and height. If the correct height is provided to the model, the CVA station shows up as visible in the model results. We have carried out this visibility comparison for a lot of other locations and came to the same good consistency as shown for the previous two examples.

By comparing the result from the measurement and the simulation in Figure B, we conclude that the visibility of model and measurements matches very well. We can also conclude that the usage of the physical Earth radius, and not its $\frac{4}{3}$ modified value, is correct to calculate the radio horizon (compare equation $\square$ in section $\nabla$ ).

\section{Signal comparison}

As the second quality criteria for the model, we compare the power of the received DME signal at the output of the aircraft antenna, between our model and the measurements. Please keep in mind that the DME software model randomly generates pulse-pairs with the statistical values from the DME standard. Thus simulated signals are obviously not the exact same as the measurement data. The important property for comparison is that the modelled DME power levels, pulse rates and ID signatures resemble the measurement data. Figure 4 shows the comparison of the re- 

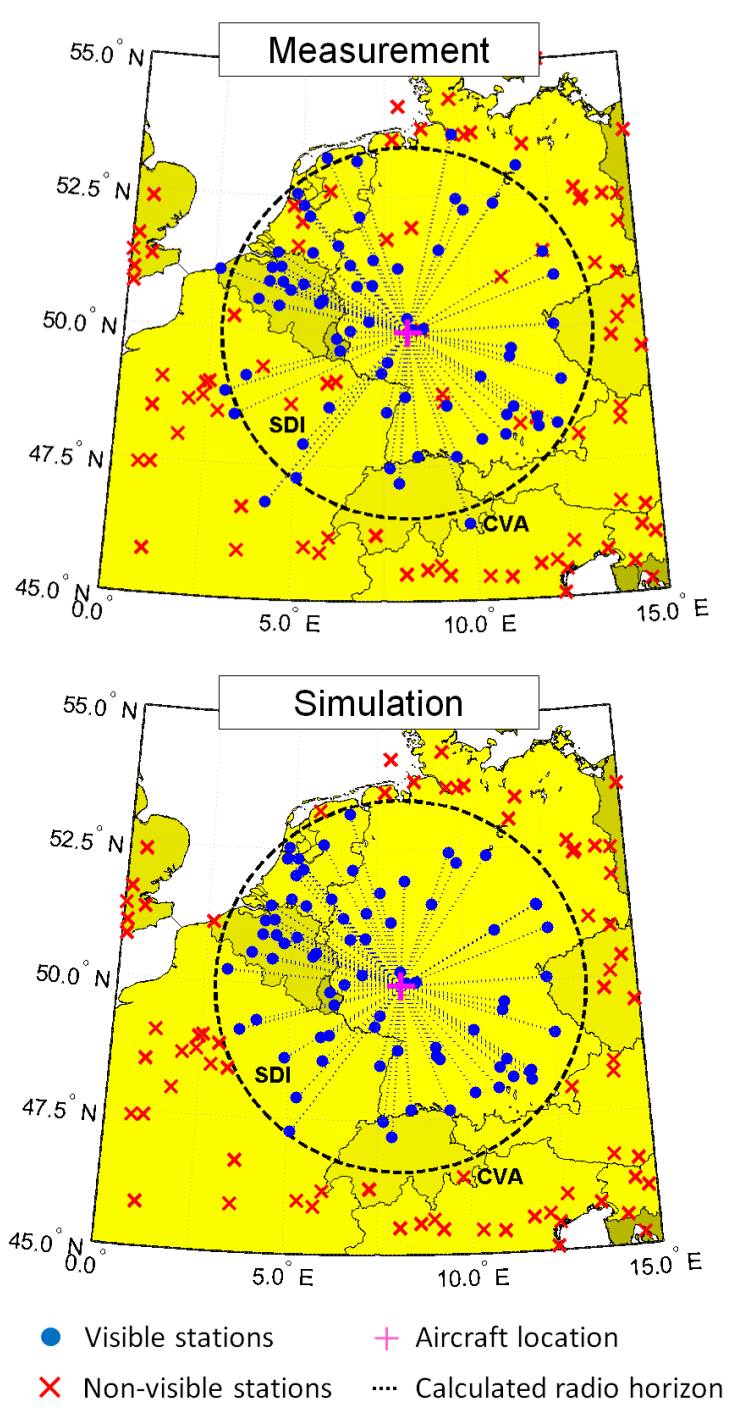

Figure 3: Comparison of the DME/TACAN ground station visibility for FL390.

ceived DME signals from the measurement data and the DME signals generated by the model. The waterfall diagram of the measured and modelled signals for the location RUDUS at flight levels FL390 and FL50 are shown in the upper and lower part of Figure 4(a) and Figure 4(b), respectively.
Power level of higher than $-66 \mathrm{dBm}$ is represented by black, while white is used for lower.

The modelled DME signal and the measured signal is very similar. For FL390, the power levels of the measured and modelled stations are showing a good match. The TACAN bursts can be well seen in both cases. For FL50, the number of visible DME stations matches perfectly. Again the power level is very similar. Since the aircraft is flying at low altitude the chance of terrain blockage is reduced. Notable comparison point is the ID sequence transmitted by the TACAN station 'BUE' at frequency $1205 \mathrm{MHz}$. The periodically transmitted ID pulse-pairs can be clearly seen in both the measured and the modelled signal. In addition to the DME signals, the secondary radar transmitted at the aircraft can be seen in the measurement data as a wide band interference (at 1.2 and $1.6 \mathrm{~ms}$ of Figure 4(a) upper part).

We conclude that also from a signal comparison point of view a very good match between model and measurement has been achieved.

\section{Conclusion}

The combined effect from aircraft antenna and fuselage has been identified as a very important part of the DME signal propagation chain, we derived an aircraft antenna diagram, that combines the effects from the GNSS antenna, receiving DME ground signals and the effects of the aircraft's fuselage. We provide this antenna diagram together with the software model. The most important result of the antenna diagram is, 

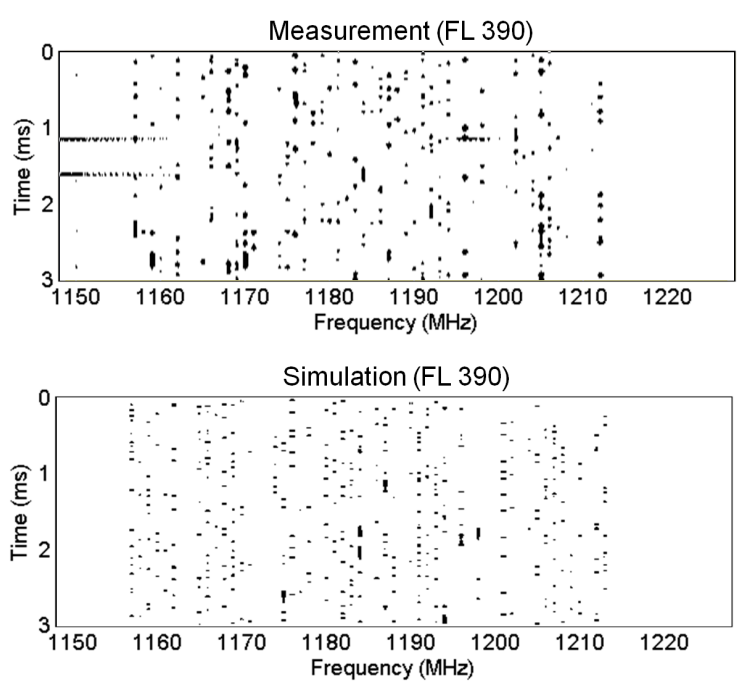

(a) FL 390
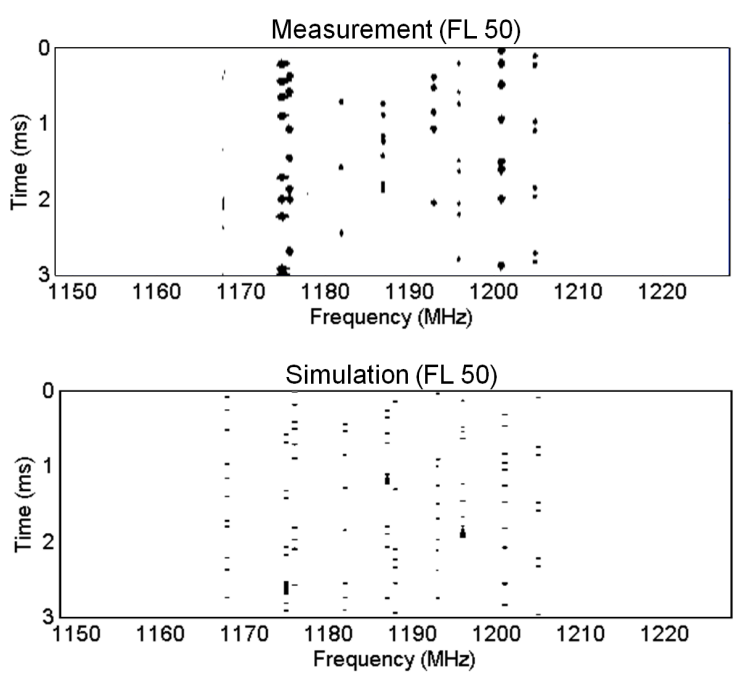

(b) FL 50

Figure 4: Comparison of the DME/TACAN signals in different flight levels between measurements and simulations at FL390 and FL50. Power level of higher than $66 \mathrm{dBm}$ is represented by black, while white is used for lower.

that it shows a major attenuation belt the antenna horizon. From the aircraft per- spective nearly all DME ground stations are located at the horizon. This leads to a strong attenuation of the received DME ground station signals if the aircraft is flying straight and levelled since the attenuation belt attenuates the DME station at the horizon. On the other hand if the aircraft is rolling (turning or pitching) the attenuation belt is rolling together with the aircraft. In this situation most of the DME ground stations at the horizon are no longer attenuated by the antenna's attenuation belt which leads to significant higher DME reception level at the aircraft. In the end the interference level on GNSS will be significantly higher when the aircraft is manoeuvring.

We compared measurements and simulations in many cases and published some examples here. In these examples the measured radio horizon matches very well with the model. In contrast to previous publications [10] which model the refraction of radio waves by enlarging the earth radius by $\frac{4}{3}$, we found that for the propagation of the DME ground station signals on carrier frequencies in L-Band the appropriate selection shall be the physical Earth radius without modifications.

The modelled power levels of the DME signals from the ground stations received at the aircraft antenna correspond well with the measurements. DME pulse rates, ID sequences and TACAN bursts of the model are matching also well with the measured signals. This good consistency of the comparisons illustrates the accuracy of the proposed model. We conclude that the model and the reality matches very well.

We provide a ready to use software model simulating DME signals received at an air- 
craft antenna. This antenna output signal can be used as an interference source to GNSS by the navigation community. In contrast to existing models, our proposed model is verified against measurements which offers a new dimension of validity. The model generates the signal of a set of DME stations calibrated to a correct power level. Thus in a navigation application the so generated signal is GNSS signaland receiver independent and can be used for a big variety of applications. Finally the modular structure of the model offers a wide rage of usage for other projects where DME stations are received either as useful signal or interference.

\section{Future Work}

In the future we intend to include terrain effects into the model for even more accurate simulations. To realize this we plan to use digital terrain models such as SRTM [4] or data from the Tandem X mission [5]. Using terrain data we will be able to predict shadowing by terrain more precisely.

Furthermore we plan to identify more interference sources that we have recorded such as primary and secondary RADAR, JTIDS and DME interference caused by the aircraft interrogating the DME ground stations.

Using the model for simulations we expect information on the maximum observed power level of a DME signal which is of interest for frequency selective interference mitigation [9], since in this mitigation concept the received signal level must stay in the linear region of the signal processing chain. Otherwise the needed FFT is im- possible.

Another important figure is the maximum observed DME pulse rate at the aircraft antenna. This pulse rate leads to a duty cycle of GNSS vs. DME signal receptions for a pulse blanking receiver. Since this receiver type is blanking the DME pulses the higher duty cycle will result to a worse equivalent $C / N_{0}$ at the pulse blanking receiver.

\section{Model Download}

A MATLAB implementation of the model can be downloaded at:

http://www.kn-s.dlr.de/satnav/. 


\section{References}

[1] Federal Aviation Administration. Aeronautical information manual. Technical report, Washington DC, USA, December 2015.

[2] World Radio Conference. Final Acts. International Telecommunication Union (ITU), Istanbul, 2000.

[3] Holmer Denks, Alexander Steingass, Achim Hornbostel, and Vincent Chopard. GNSS receiver testing by hardware simulation with measured interference data from flight trials. In Proceedings of the Institute of navigation conference ION GNSS, Savanna, GA, USA, September 2009.

[4] DLR. SRTM Data access - EOWEB https://centaurus.caf.dlr.de: 8443/, 2016. [Online].

[5] DLR. New 3d world map tandem-x global elevation model completed http://www.dlr.de/ dlr/en/desktopdefault.aspx/ tabid-10081/151_read-19509/\#/ gallery/24516, 2017. [Online].

[6] Fabio Dovis, Luciano Musumeci, and Jaron Samson. Performance comparison of transformed-domain techniques for pulsed interference mitigation. Proceedings of the 25th International Technical Meeting of The Satellite Division of the Institute of Navigation (ION GNSS 2012), pages 35303541, September 2012.

[7] EUROCAE. ED-54: Minimum operational performance requirements for distance measuring equioment interrrogator (DME/N and DME/P) operating within the radio frequency range 960 to $1215 \mathrm{MHz}, 1987$.

[8] EUROCAE. Minimum operational performance specification for airborne open service galileo satellite receiving equipment. Technical report, EUROCAE, Brussels, Belgium, June 2013.

[9] Grace Xingxin Gao, Liang Heng, Achim Hornbostel, Holmer Denks, Michael Meurer, Todd Walter, and Per Enge. DME/TACAN interference mitigation for GNSS: Algorithms and flight test results. GPS Solutions, 17(4):561-573, 2013.

[10] Abdollah Ghasemi, Ali Abedi, and Farshid Ghasemi. Propagation Engineering in Wireless Communications. Springer, Heidelberg, Germany, 2012. ISBN 978-1-4614-1076-8.

[11] Abdollah Ghasemi, Ali Abedi, and Farshid Ghasemi. Propagation Engineering in Radio Links Design. Springer Publishing Company, Incorporated, 2013.

[12] Joseph Grabowski and Christopher Hegarty. Characterization of L5 receiver performance using digital pulse blanking. Proceedings of the 15th International Technical Meeting of the Satellite Division of The Institute of Navigation (ION GPS 2002), pages 1630 - 1635, September 2002.

[13] Marcos Heckler. Performance of antenna arrays for satellite navigation 
and communication installed on aircraft. $8^{\text {th }}$ ONERA-DLR Aerospace Symposium ("ODAS" 2007), pages 10pp. CD-ROM, October 2007.

[14] Marcos Heckler, Lukasz Greda, and Achim Dreher. Analysis of a navigation antenna installed on a civil airplane. IEEE Antennas Propagation Society International Symposium, pages 4pp. CD-ROM, July 2008.

[15] Christopher Hegarty and A.J. Van Dierendonck. Civil GPS/WAAS signal design and interference environment at t1176.45 MHz: Results of RTCA SC159 WG1 activities. Proceedings of the 12th International Technical Meeting of the Satellite Division of The Institute of Navigation (ION GPS 1999), pages 1727-1736, September 1999.

[16] Christopher Hegarty, A.J. Van Dierendonck, Dan Bobyn, Michael Tran, and Joe Grabowski. Suppression of pulsed interference through blanking. Proceedings of the IAIN World Congress and the 56th Annual Meeting of The Institute of Navigation, pages 399-408, January 2000.

[17] Christopher Hegarty, Taehwan Kim, Swen Ericson, Patrick Reddan, Thomas Morrissey, and A.J. Van Dierendonck. Methodology for determining compatibility of GPS L5 with existing systems and preliminary results. Proceedings of the 55th Annual Meeting of The Institute of Navigation, pages 399-408, June 1999.

[18] KATHREIN-Werke KG. Technical description for DME omnidirectional gain antenna, 1986. Model FAN 86 Code 9038960.

[19] Sherman C. Lo, Per K. Enge, and Mitchell J. Narins. Design of a passive ranging system using existing distance measuring equipment (DME) signals \& transmitters. NAVIGATION, Journal of The Institute of Navigation, 62 No. 2:131-149, 2015.

[20] L. Musumeci, J. Samson, and F. Dovis. Experimental assessment of distance measuring equipment and tactical air navigation interference on GPS L5 and galileo E5a frequency bands. In Satellite Navigation Technologies and European Workshop on GNSS Signals and Signal Processing, (NAVITEC), 2012 6th ESA Workshop on, pages 1-8, Dec 2012.

[21] National Aerospace Laboratory NLR. MOPS GNSS Aviation standard draft 2 version $2.1(9 / 10 / 2014)$. Technical report, National Aerospace Laboratory NLR, Amsterdam, Netherlands, October 2014 .

[22] International Civil Aviation Organization. Communications database. http://www.icaodata.com/ http://goo.gl/phUvRe.

[23] International Civil Aviation Organization. Aeronautical Telecommunications - ICAO Annex 10 - Volume 1 - Aeronautical Telecommunications Radio Navigation Aids. Technical report, ICAO, Montréal, Canada, 2012.

[24] John Owen. Results of an investigation into the use of $1175 \mathrm{MHz}$ and 
$1202 \mathrm{MHz}$ for GNSS signals in european airspace. Proceedings of the 2002 National Technical Meeting of The Institute of Navigation, January 2002.

[25] EU Project. Airborne new and advanced satellite techniques \& technologies in a system integrated approach. http://goo.gl/w9WuC6. Accessed: 2016/06/29.

[26] EU STANDARDS Project. Interim gnss antenna mops. goo.gl/kZo721, January 2014. [Online].

[27] Mathieu Raimondi, Christophe Macabiau, and Olivier Julien. Frequency domain adaptive filtering against pulsed interference: Performance analysis over europe. Proceedings of the 2008 National Technical Meeting of The Institute of Navigation, pages 164-176, September 2008.

[28] B. Roturier. Report on DME interference on GPS/L5. Technical report, EUROCONTROL, Brussels, Belgium, 1999.

[29] A. Simsky, W. De Wilde, T. Willems, D. Mertens, E. Koitsalu, and J-M. Sleewaegen. First field experience with L5 signals: DME interference reality check. Proceedings of the 22nd International Technical Meeting of The Satellite Division of the Institute of Navigation (ION GNSS 2009), pages 29-37, September 2009.

[30] Alexander Steingass. Analysis of DME/TACAN Interference on the Lower L-Band. Proceedings of the 26th
International Technical Meeting of The Satellite Division of the Institute of Navigation (ION GNSS+ 2013), pages 3409-3416, September 2013.

[31] International Telecommunication Union. Recommendation ITU-R-P528 propagation curves for aeronautical mobile and radio navigation services using the VHF, UHF and SHF bands. Technical report, ITU, Geneva, 2012.

[32] E.W. Weisstein. CRC Concise Encyclopedia of Mathematics, Second Edition. CRC Press, 2002.

[33] "EUROCONTROL WP". Liaison statement to GNSS-P. https://goo. gl/MGyfL9, 1999. 